\title{
VR and HCI Labs at the Czech Technical University in Prague
}

\author{
Roman Berka, Jiří Bittner, Pavel Slavík \\ Department of Computer Graphics and Interaction \\ Faculty of Electrical Engineering \\ Czech Technical University in Prague \\ Karlovo namesti 13, 12135 Praha 2 \\ Czech Republic \\ WWW: http://dcgi.fel.cvut.cz
}

\begin{abstract}
This paper describes the mission, objectives, and research directions of the Department of Computer Graphics and Interaction of the Czech Technical University in Prague. It also gives a brief overview of the running research projects and collaborations.

Index Terms-virtual reality; human computer interaction; computer graphics.
\end{abstract}

\section{HISTORY AND MISSION}

The Department of Computer Graphics and Interaction (DCGI) is a part of the Faculty of Electrical Engineering of the Czech Technical University in Prague (CTU). DCGI was founded in 2008 by members of Computer Graphics Group which was formed in 1992 at the Department of Computer Science and Engineering. The research activities of DCGI focus on two main directions - Computer Graphics and Human-Computer Interaction. These two research directions are developed within two special laboratories: Virtual Reality Laboratory (VRLab) and Usability Laboratory (ULab). In addition a special institute - Institute for Intermedia (IIM) - is also run by the department. All these facilities serve both for research and education at the CTU. The education part covers $\mathrm{BSc}, \mathrm{MSc}$, and $\mathrm{PhD}$ study programmes. In the BSc study track DCGI offers 10 courses in the both fields (Computer Graphics and $\mathrm{HCI}$ ), in the MSc study track it offers 12 courses. DCGI currently employs 14 academic staff members and $13 \mathrm{PhD}$ students.

The long term objective of our department is to reach and maintain the level of leading European universities in both research and education activities. In particular we aim:

- to educate bachelor, master, and doctoral students to become graduates with a high competence and great potential for their future careers.

- to actively participate in the current research in the fields of Computer Graphics and Human-Computer Interaction.

- to publish at reputable international conferences and prestigious journals.

- to support teaching and research by carrying out projects funded by grant agencies and important commercial organizations.

- to collaborate with Czech and foreign partners in research and teaching.

\section{OBJECTIVES AND RESEARCH LINES}

In this section we describe the activities of the three main labs of DCGI: Virtual Reality Lab, Usability Lab and the Institute of Intermedia (see Figure 1).

\section{A. Virtual Reality Lab}

The virtual reality laboratory (VRLab) is the main lab of the computer graphics group at DCGI. VRLab contains equipment for experiments in virtual and augmented reality as well as tests of advanced rendering and interaction techniques. In particular it has at disposal a stereoscopic projection wall, a tracking system, and two types of augmented reality setups.

The members of computer graphics group conduct active research in the field of real-time and realistic rendering (global illumination, visibility computations) [1], [2], [3], [4], [5], [6], [7], [8], advanced data structures for computer graphics [9], [10], computer games [11], and also in the field of content creation and 3D reconstruction for images and video [12], [13], [14], [15]. The computer graphics group has intensive links to several research groups in Europe (Vienna University of Technology, Max Planck Institute for Informatics, University of Girona) as well as overseas (Purdue University, Arizona State University). More information about VRLab can be found at http://dcgi.felk.cvut.cz/en/research.

\section{B. Usability Lab}

The Usability Lab (ULab) of DCGI has been established in 2004 in cooperation with Sun Microsystems. At that time it was the very first professionally equipped usability lab in the country. The main objective of the laboratory is to perform usability research in the framework of projects performed at CTU (in the past mainly in cooperation with Sun Microsystems). As the CTU students traditionally participate during their study in research projects of various type, the lab is thus used also in education and students get the opportunity of acquaintance with usability lab and with methodology used in usability testing. As a result the graduates from CTU have the basic knowledge in usability testing plus the knowledge about the methodology of user interface (UI) design. More details can be found in [16]. 


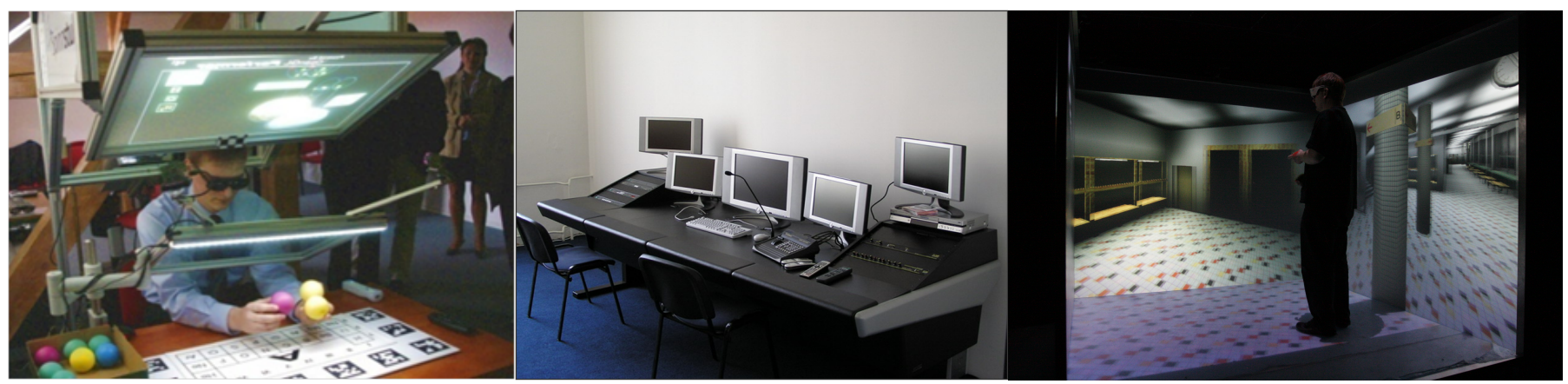

Fig. 1. Overview of the DCGI labs: (left) Augmented reality setup at the Virtual Reality Lab, (center) the Usability Lab, (right) CAVE at the Institute of Intermedia.

We focus on two main research lines: UI for mobile computing and UI for handicapped users (accessibility issues). In the field of mobile computing we pay a special attention to issues in graphical interaction in mobile environment (small screen, multimodal interaction, role of context etc.). In the second field we investigate namely the needs of blind and visually impaired users. Technically speaking our HCI research has two foci: the design and the consequent testing of the UI developed [17], [18], [19], [20]. More information about ULab can be found at http://www.ulab.cz.

\section{Institute of Intermedia}

The Institute of Intermedia (IIM) has been established in 2007 as a cooperative platform by three universities: Czech Technical University in Prague (CTU), Academy of Performing Arts in Prague (APA) and Academy of Arts, Architecture and Design in Prague.

The goal of the IIM is to create a common platform for students of art and technical disciplines, scientists and freelance artists to cooperate on common projects and experiments. The activities of IIM cover the fields of virtual reality, multimedia, stereoscopy, light design, and audio-visual performances. The main objective of IIM and its founders is to support the symbiosis of humanistic oriented disciplines with the technical sciences. We expect that this interdisciplinary relation boosts a creative potential of all participating students, researchers, and artists. The main three tracks of IIM activities are thus formed by education, research and public performances with exhibitions. The performances are typically realized by our students supported by freelance artists cooperating with IIM.

The research activities of IIM are in its initial phase. We build on our experience from the area of virtual reality and stereoscopy and on the availability of advanced hardware setups such as CAVE system and optical motion tracking. Our first attempts lie in explorations of collaborative interaction in distributed virtual environments. Our goals are directed to intelligent multimedia communication methods allowing adaptation of data transmitted among VR devices with different level of immersion. This technology should make possible to exchange multimedia and 3D content together with meta data independently of the abilities of the particular devices. For example we develop a methodology to observe a session of two CAVE-like devices by a user with a notebook, while maximizing the throughput of the available information transfer to the user [21]. More information about IIM can be found at http://www.iim.cz.

\section{Research projects}

DCGI participates in both national and international research projects dealing with computer graphics and human computer interaction. In this section we list the currently running projects. Some of our recent research results are depicted in Figure 2.

a) VERITAS: The VERITAS project aims to develop, validate and assess tools for built-in accessibility support at all stages of product development, including specification, design, development and testing. The goal is to introduce simulation based and virtual reality testing at all stages of assistive technologies product design and development into various applications that handicapped users can use. The VERITAS project is funded from the 7th Framework Programme of the European Commission. More information about the project can be found at http://veritas-project.eu.

b) AEGIS: The AEGIS project seeks to determine whether 3rd generation access techniques will provide a more accessible, more exploitable and deeply embeddable approach in the mainstream desktop, rich internet and mobile applications. The AEGIS project is funded from the 7th Framework Programme of the European Commission. More information about the project can be found at http://www.aegis-project.eu.

c) ACCESSIBLE: The ACCESSIBLE project will exploit the technologies behind the recent expansion of accessibility tools and standardization methodologies, in order to provide an integrated simulation assessment environment for supporting the production of accessible software applications. The ACCESSIBLE project is funded from the 7th Framework Programme of the European Commission. More information about the project can be found at http://www.accessible-eu.org.

d) Center for Computer Graphics (CPG): CPG is s common research project of four universities from the Czech Republic. The aim of the project is to strengthen the research potential of the partner institutions by bringing their experts together and providing a platform for financing their research projects in the field of advanced computer graphics techniques. 

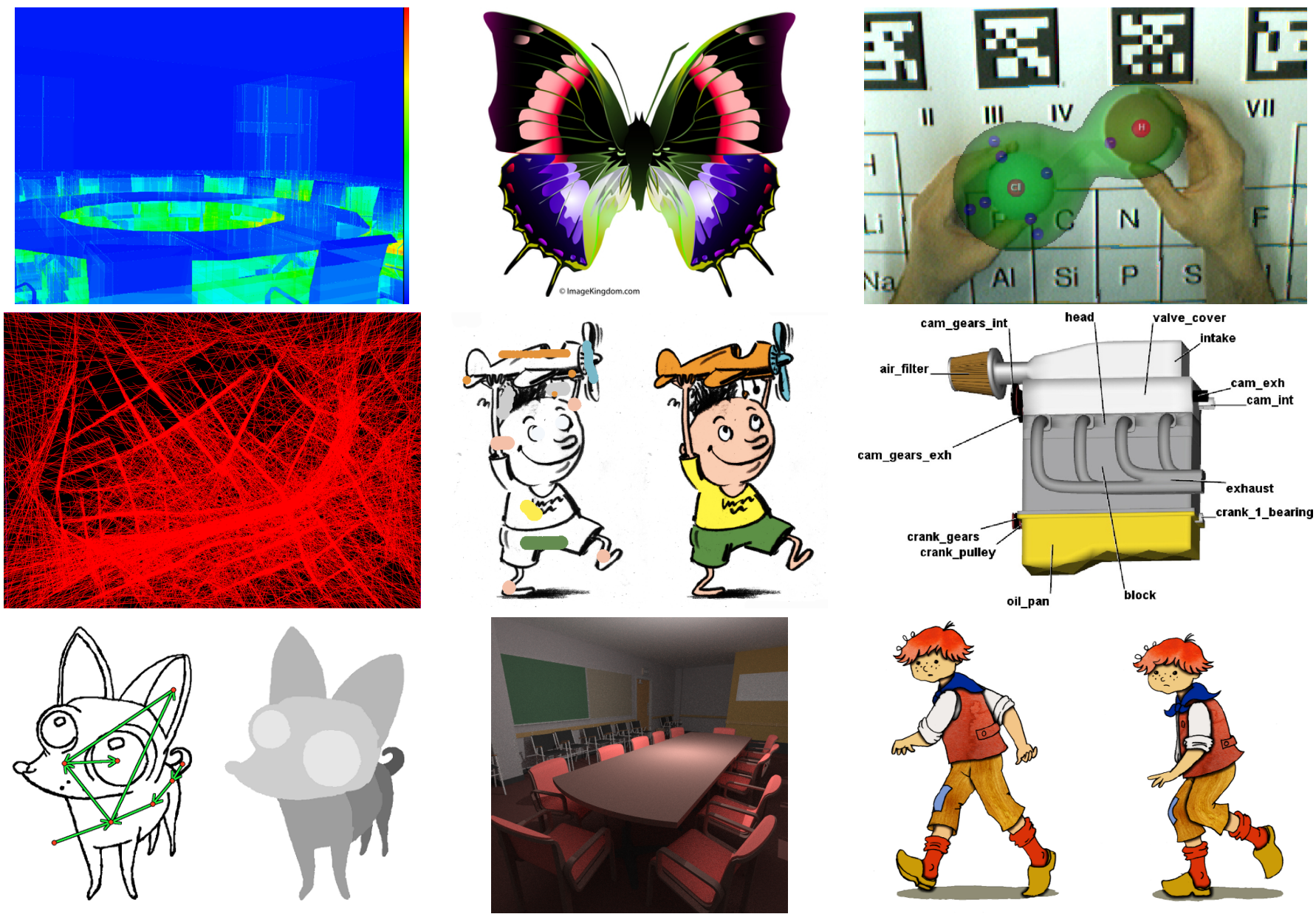

Fig. 2. Images illustrating some of the results of our recent research projects (all images are a courtesy of the authors of the corresponding papers). (top) Ray Tracing with Sparse Boxes [8], Perceptual Evaluation of Color-to-Grayscale Image Conversions [4], Real-time Color Ball Tracking for Augmented Reality [22]. (middle) Adaptive Global Visibility Sampling [2], LazyBrush: Flexible Painting Tool for Hand-drawn Cartoons [23], Layout-aware optimization for interactive labeling of 3D models, (bottom) Adding Depth to Cartoons Using Sparse Depth (In)equalities [13], Efficient Stack-less Traversal Algorithm for Ray Tracing with BVH [9] (the depicted model is a courtesy of Anat Grynberg and Greg Ward), TexToons: Practical Texture Mapping for Hand-drawn Cartoon Animations [12].

e) ARGIE: Global Illumination for Augmented Reality in General Environments (ARGIE) is a research project of the computer graphics group. The project aims at computing realistic images mixing a real scene with virtual objects while using only a very limited amount of a priori information about the real scene. Our project aims at designing methods and algorithms for computing different global illumination components in augmented reality including illumination effects like shadows, diffuse, glossy and specular reflections and caustics.

f) CAVE to CAVE: CAVE to CAVE (C2C) is a project realized in cooperation with the Czech Internet provider CESNET where the main goal is the development of alternative ways to transmit content presented in one CAVE device to another distant visual device using broad-band networks.

g) Stereo: Stereo is a project supported by Czech Ministry of Education oriented to development of stereoscopy production chain usable in education process and later also in research. The project is realized in cooperation with Film and TV Faculty of Academy of Performing Arts in Prague. h) Digitization of the Langweil Model: The Langweil model of Prague is a historical coloured paper model of the Prague city center from the first half of the 19th century. Our group participated in the project of digitizing the model, which was financed by the Prague City Museum. One of the research oriented outcomes of the project is a challenging data set for various computer vision algorithms available for download after registration at http://dcgi.felk.cvut.cz/langweil3d. More details about the project can be found at [24].

i) VRUT: The VRUT (Virtual Reality Universal Toolkit) project is developed in cooperation with the Skoda-Auto company. It aims at the development of various tools for high quality stereoscopic presentations and evaluations of the virtual car prototypes. In the project we also develop a driving simulation software designed primarily for human-machine interface evaluation.

\section{CONCLUSION}

The above described labs were established with the aim to support both the research and educational activities of our 
department. Our experience gained in last few years has shown that these labs opened up new prospects in two main areas: (1) Students got access to sophisticated technologies and learned how to operate them. This fact will improve their position on labor market after their graduation from CTU. (2) It has been possible to get involved in international research projects and national industrial projects where the equipment of these labs is used.

The labs created a synergy where mutual crossfertilization between Computer Graphics, HCI, and art is taking place. Many new interesting ideas have appeared and they were brought to life in the projects performed in these labs. We hope that the activities of our labs will successfully continue in the future and one of our aims is to extend our activities by involving new people and new collaborating institutions.

\section{ACKNOWLEDGMENTS}

Our research activities are partially supported by the MSMT under the research programs LC-06008 (Center for Computer Graphics) and MSM 6840770014, by the Grant Agency of the Czech Republic under projects number P202/11/1883 (ARGIE) and by the European Commission under project number FP7-ICT-247765 (VERITAS), FP7-ICT224348 (AEGIS), FP7-ICT-224145 (ACCESSIBLE).

\section{REFERENCES}

[1] V. Havran, J. Filip, and K. Myszkowski, "Bidirectional texture function compression based on multi-level vector quantization," Computer Graphics Forum, vol. 29, no. 1, pp. 175-190, 2010.

[2] J. Bittner, O. Mattausch, P. Wonka, V. Havran, and M. Wimmer, "Adaptive global visibility sampling," ACM Trans. Graph., vol. 28, pp. 94:1-94:10, July 2009.

[3] V. Gassenbauer, J. Krivanek, and K. Bouatouch, "Spatial directional radiance caching," Computer Graphics Forum, vol. 28, no. 4, pp. 11891198, 2009.

[4] M. Cadik, "Perceptual evaluation of color-to-grayscale image conversions," Computer Graphics Forum, vol. 27, no. 7, pp. 1745-1754, 2008

[5] J. Obert, J. Krivanek, F. Pellacini, D. Sykora, and S. Pattanaik, "icheat: A representation for artistic control of indirect cinematic lighting," Computer Graphics Forum, vol. 27, no. 4, pp. 1217-1223, 2008.

[6] O. Mattausch, J. Bittner, and M. Wimmer, "Chc++: Coherent hierarchical culling revisited," Computer Graphics Forum, vol. 27, no. 2, pp. 221-230, 2008.

[7] R. Herzog, V. Havran, S. Kinuwaki, K. Myszkowski, and H.-P. Seidel, "Global illumination using photon ray splatting," in Computer Graphics Forum, vol. 26(3), no. 3, Prague, Czech Republic, 2007, pp. 503-513.

[8] V. Havran and J. Bittner, "Ray tracing with sparse boxes," in Proceedgings of Spring Conference on Computer Graphics, 2007, pp. 49-54.
[9] M. Hapala and V. Havran, "Review: Kd-tree Traversal Algorithms for Ray Tracing," Computer Graphics Forum, vol. 30, no. 1, pp. 199-213, march 2011.

[10] M. Hapala, T. Davidovič, I. Wald, V. Havran, and P. Slusallek, "Efficient stack-less traversal algorithm for ray tracing with bvh," in Proceedgings of Spring Conference on Computer Graphics, 2011, pp. 29-34.

[11] L. Kavan, S. Collins, J. Žára, and C. O'Sullivan, "Geometric skinning with approximate dual quaternion blending," ACM Trans. Graph., vol. 27, pp. 105:1-105:23, November 2008.

[12] D. Sýkora, M. Ben-Chen, M. Čadík, B. Whited, and M. Simmons, "Textoons: Practical texture mapping for hand-drawn cartoon animations," in Proceedings of International Symposium on Non-photorealistic Animation and Rendering, 2011, pp. 75-83.

[13] D. Sýkora, D. Sedlacek, S. Jinchao, J. Dingliana, and S. Collins, "Adding depth to cartoons using sparse depth (in)equalities," Computer Graphics Forum, vol. 29, no. 2, pp. 615-623, 2010.

[14] L. Cmolik and J. Bittner, "Layout-aware optimization for interactive labeling of 3d models," Computers \& Graphics, vol. 34, no. 4, pp. 378 - 387, 2010, procedural Methods in Computer Graphics; Illustrative Visualization

[15] Z. Mikovec, P. Slavik, and J. Zara, "Cultural heritage, user interfaces and serious games at CTU prague," in Virtual Systems and Multimedia, 2009. VSMM '09. 15th International Conference on, sept. 2009, pp. 211 $-216$.

[16] P. Slavík and Z. Míkovec, "Usability in the Czech Republic," in Global Usability, ser. Human-Computer Interaction Series. Springer London, 2011, pp. 137-152.

[17] L. Kunc, J. Kleindienst, and P. Slavík, "Talking head as life blog," in Proceedings of the 11th international conference on Text, Speech and Dialogue, ser. TSD '08. Berlin, Heidelberg: Springer-Verlag, 2008, pp. 365-372.

[18] I. Maly, J. Curin, J. Kleindienst, and P. Slavik, "Creation and visualization of user behavior in ambient intelligent environment," in Information Visualisation, 2008. IV '08. 12th International Conference, 2008, pp. 497 -502 .

[19] O. Poláček, A. J. Sporka, and P. Slavík, "Music alphabet for lowresolution touch displays," in Proceedings of the International Conference on Advances in Computer Enterntainment Technology, ser. ACE '09. New York, NY, USA: ACM, 2009, pp. 298-301.

[20] O. Poláček and Z. Míkovec, "Hands free mouse: comparative study on mouse clicks controlled by humming," in Proceedings of the 28th of the international conference extended abstracts on Human factors in computing systems, ser. CHI EA '10. New York, NY, USA: ACM, 2010, pp. 3769-3774

[21] Z. Trávníček and R. Berka, "Multi-threaded real-time video grabber," in WSCG'10 Communication Papers Proceedings, 2010.

[22] D. Sýkora, D. Sedláček, and K. Riege, "Real-time color ball tracking for augmented reality," in Proceedgings of Eurographics Symposium on Virtual Environments, 2008, pp. 9-16.

[23] D. Sýkora, J. Dingliana, and S. Collins, "Lazybrush: Flexible painting tool for hand-drawn cartoons," Computer Graphics Forum (Proceedings of Eurographics 2009), vol. 28, no. 2, pp. 599-608, 2009.

[24] D. Sedlacek and J. Zara, "Graph cut based point-cloud segmentation for polygonal reconstruction," in Proceedings of the 5th International Symposium on Advances in Visual Computing: Part II, ser. ISVC '09. Berlin, Heidelberg: Springer-Verlag, 2009, pp. 218-227. 